\author{
386684 \\ DPSP-83-1-4 (Excerpts)
}

\title{
TECHNICAL REPORT FOR APRIL 1983 (EXCERPTS)
}

by

Dupont SRP

This is a Technical Report

This paper was prepared in connection with work done under Contract No. DE-AC09-89SR18035 with the U.S. Department of Energy. By acceptance of this paper, the publisher and/or recipient acknowledges the U.S. Government's right to retain a nonexclusive, royalty-free license in and to any copyright covering this paper, along with the right to reproduce and to authorize others to reproduce all or part of the copyrighted paper.

RECORDS ADMINISTRATION

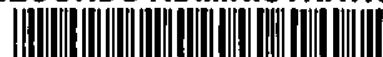

R1705506 


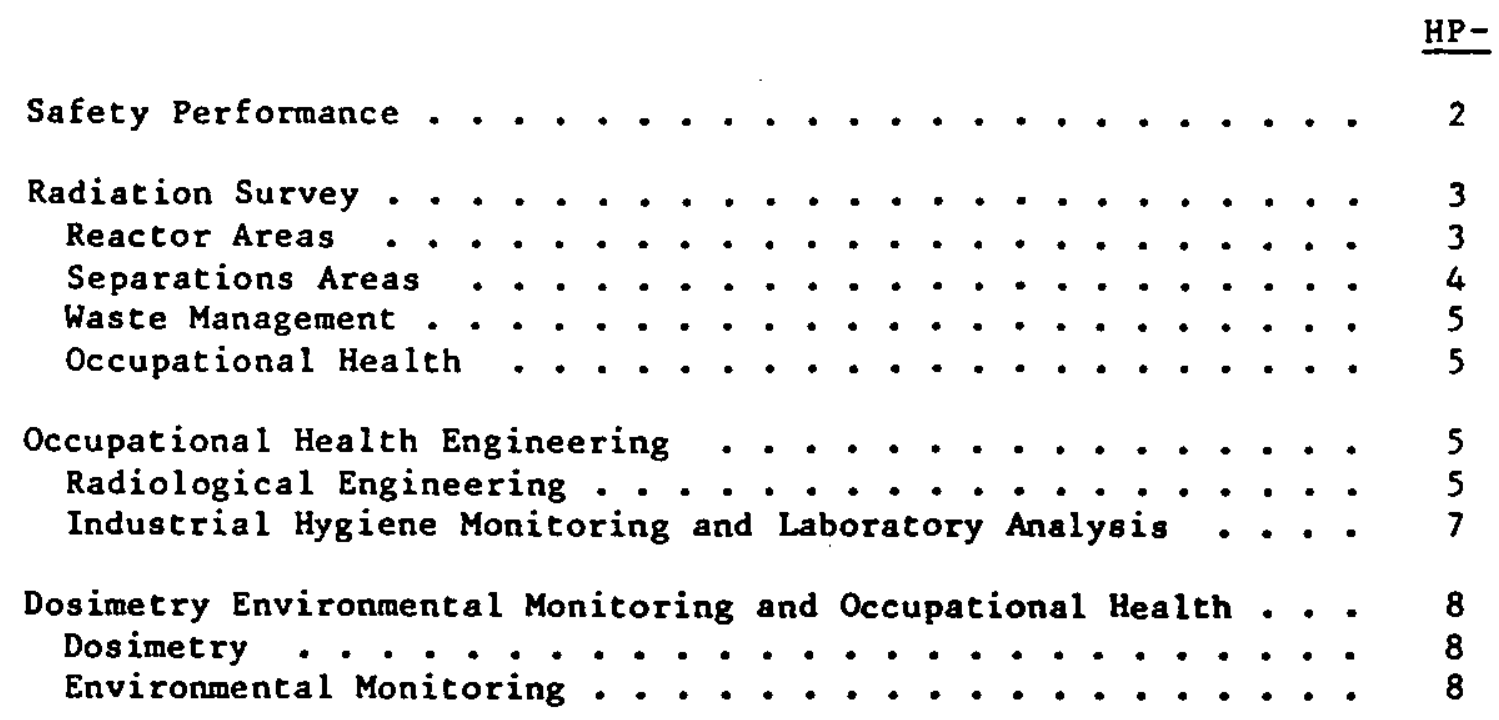




\title{
RADIATION SURVEY
}

\author{
Reactor Areas
}

P AREA

Whole body radiation exposure rates to $10 \mathrm{R} / \mathrm{hr}$ and extremity rates to $20 \mathrm{R} / \mathrm{hr}$ were encountered during removal of a nose plug for inspection of the reactor tank exterior wall during a scheduled outage. A radiation exposure rate of 50 $\mathrm{R} / \mathrm{hr}$ was measured flush with the biological shield opening. Transferable contamination to $2.0 \times 10^{4} \mathrm{c} / \mathrm{m}$ beta-gamma/0.1 $\mathrm{m}^{2}$ was controlled by the use of a containment hut. Special TLD dosimeters were worn by personnel to audit exposure to whole body, head, hands, and arms. An estimated radiation dose totaling 2.2 rems was accumulated.

Radiation exposure rates to $100 \mathrm{mR} / \mathrm{hr}$ and airborne tritium concentrations to $2.0 \times 10^{-5} \mu \mathrm{Ci} / \mathrm{cc}$ were measured during regasketing of heat exchanger inhibitor heads. Personnel involved in the work received an estimated exposure of 2.3 rems.

The maximum tritium assimilation during the report period was $7.3 \mathrm{uCi} / 1$ of body fluids. The assimilation occurred when a distillation area sightglass leaked and a Reactor employee came in contact with moderator. Airborne tritium concentrations to $1.0 \times 10^{-4} \mathrm{\mu Ci} / \mathrm{cc}$ were present. Air-supplied plastic suits were worn to repair the sightglass.

L AREA

Process area radiation exposure rates for renovation work ranged from 1 to 5 $\mathrm{mR} / \mathrm{hr}$. An estimated $10,000 \mathrm{gal}$ of virgin moderator was added to the reactor system in preparation for leak checking and flushing. Airborne tritium concentrations averaged less than $1.0 \times 10^{-5} \mathrm{Hi} / \mathrm{cc}$ and no unusual radiological control problems were encountered.

\section{K AREA}

The reactor was shutdown for replacement of the top and bottom biological shield pump. Radiation exposure rates averaged $45 \mathrm{mR} / \mathrm{hr}$, and no significant airborne tritium radioactivity was encountered. A total radiation dose of 0.6 rem was accumulated by nine personnel.

\section{AREA}

No significant radiological problems occurred during the report period.

M AREA

No unusual radiation or contamination problem were encountered during the report period. 
Waste Management

BUILDING 241-F, H-AREA GENERAL

The new shielded crane was used for nine jobs involving removal of cell covers from diversion boxes and pump pits. Exposure dose rates in the cab did not exceed $1 \mathrm{mR} / \mathrm{hr}$. Radiation intensities over the open cell ranged to $1 \mathrm{R} / \mathrm{hr}$. Seventy personnel received an estimated total dose of 1.6 rems.

Radiation exposure dose rates over open risers to remove or install various jet assemblies were reduced from a maximum of $50 \mathrm{R} / \mathrm{hr}$ to $500 \mathrm{mR} / \mathrm{hr}$ by

extensive lead shielding. The total estimated dose for 67 personnel was 3.5 rems .

\section{Occupational Health}

L AREA

Airborne concentrations of xylene to $400 \mathrm{ppm}$ ( TLV $=100 \mathrm{ppm}$ ) were measured during painting of floors and walls in the process areas with "Amercoat." Personnel applying the paint wore appropriate respirators.

BUILDING 241-TANK 48

Extensive industrial hygiene monitoring continued during the salt decontamination demonstration program. Analyses of 110 air samples indicated benzene concentrations ranging to $15 \mathrm{ppm}$ in the tank purge exhaust and $<1$ ppm in personnel breathing zones ( TLV $=10 \mathrm{ppm}$ ). No additional occupational health controls were required beyond those established for the initial startup of the demonstration program.

\section{OCCUPATIONAL HEALTH ENGINEERING}

Radiological Engineering

\section{DEFENSE WASTE PROCESS ING FACILITY (DWPF)}

Unshielded glass waste canister radiation levels vs. distance were determined for four cases reflecting variable glass density and gama source term.

HIGH LEVEL WASTE SUPERNATE PROCESSING

Health Protection considerations were reviewed for the revised Z-Area layout and mode of operation. 
INDUSTR IAL HYGIENE MONITORING AND LABORATORY ANALYSES

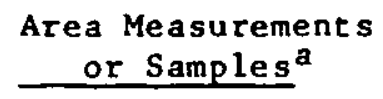

\section{Analvsis}

IH Lab

Analyses
Offplant Lab Analyses

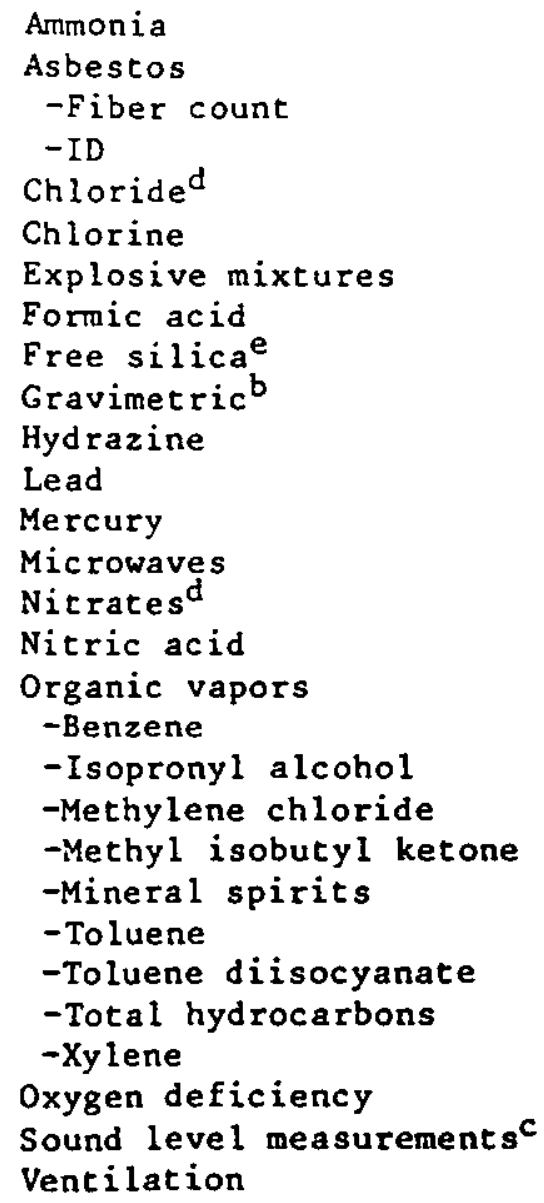

15 $--4$

30

$--$

$--$

$-$

$--$

27

$--$

$-$

$-$

$-$

\footnotetext{
a Represents 636 surveys.

bepresents 20 breathing air surveys(three samples/survey)and one oil mist survey.

${ }^{c}$ Does not include department annual surveys.

dAnalysis performed to validate new IH procedures.

e Two samples split for quality control check.
} 
SRP's Environmental Monitoring group. In general, the Georgia data agreed with SRP data even though the sample dates and collection frequencies were different in many cases. This indicates good sample collection and analyses techniques by both groups.

The GDNR environmental surveillance program near SRP consists of the following:

\section{Type Sample}

TLD's

Surface water

Groundwater

Soil

Grass

Air

Milk

Fish, wildlife, animals, fruits, vegetables, and

river sediment

\begin{tabular}{ll} 
No. of Sampling & Sampling \\
Locations & Frequency \\
\hline
\end{tabular}

\section{5}

7

10

8

8

3

7

(Analyzed when available)
Quarterly
$" 1$
$" 1$
"
Monthly

A direct comparison of GDNR data with SRP data was difficult in many cases because the exact location of the GDNR sample site was not specified. GDNR used general location categories rather than specific sample locations.

TLD data from Georgia's "near site background" and "indicator" locations were compared with SRP data from 25 -nile radius locations (table HP-8). "Near site background" locations are stations within 30 miles of the SRP that are not expected to be affected by SRP operations, whereas "indicator" locations are around the SRP plant perimeter and might be affected by plant operations. GDNR TLD measurements were approximately $60 \%$ lower than SRP's TLD measurements. The exact reason for this discrepancy is not known but may be attributed to the location of the dosimeters or the type of dosimeter. This specific information was not given in the GDNR report. GDNR indicated that they plan to correct the reported TLD measurements.

GDNR data for tritium in surface water in Port Wentworth drinking water and in milk samples from dairies near SRP were also compared with SRP results (tables HP-9 through HP-11). These data showed good agreement with each other. In many cases, the Georgia data were derived from samples supplied to GDNR by SRP.

A special survey of the Savannah River was conducted by GDNR on February 23, 1981. Water samples were collected at nine locations along the river between the New Savannah Bluff Lock Dam and Highway 301 (figure HP-2). These samples were analyzed for tritium. SRP's tritium results for comparable river samples were in good agreement with the Georgia data (table HP-12). 
The source of the mercury is being investigated by Waste Management

Technology. Samples from the contributing streams to the seepage basin are being analyzed for mercury to determine the source.

\section{RADIOACTIVE RELEASES}

No annual guides were exceeded during February. One annual guide was exceeded. during the same period in 1982 .

Also, during February, seven prorated annual guides were exceeded. Table HP-2l contains graphic summaries of the releases that have exceeded prorated annual guides. The reasons for the guides being exceeded are given below each graph.

\section{SAVANNAH RIVER}

Transport of tritium in the river is shown in table HP-17.

MERCURY RELEASES

Mercury releases to separations areas seepage basins are sumarized in table $\mathrm{HP}-18$.

\section{FECAL COLIFORM BACTERIA}

Fecal coliform bacteria data are summarized in table HP-19.

\section{RADIOACTIVE RELEASE TABLES}

Detailed radioactive release data are contained in the following tables:

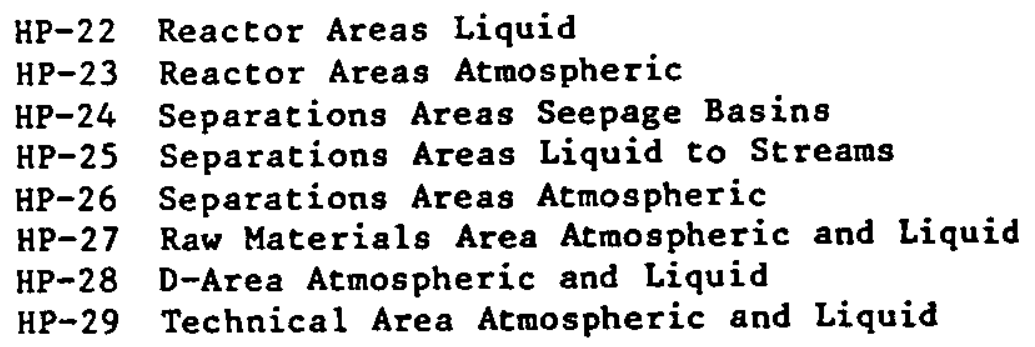




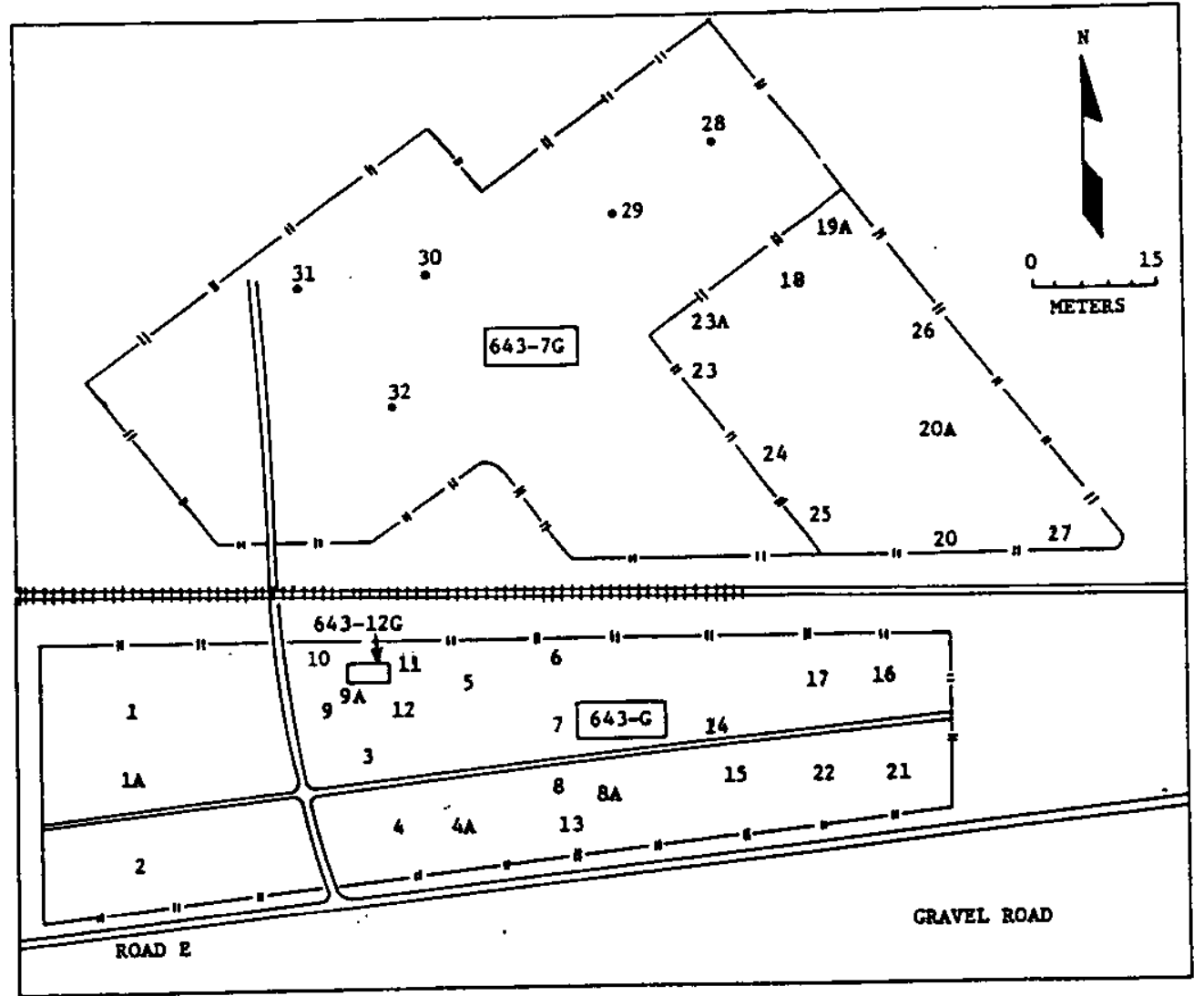

FIGURE HP-1. VEGETATION SAMPLING LOCATIONS

IN SOLID WASTE STORAGE FACILITY 


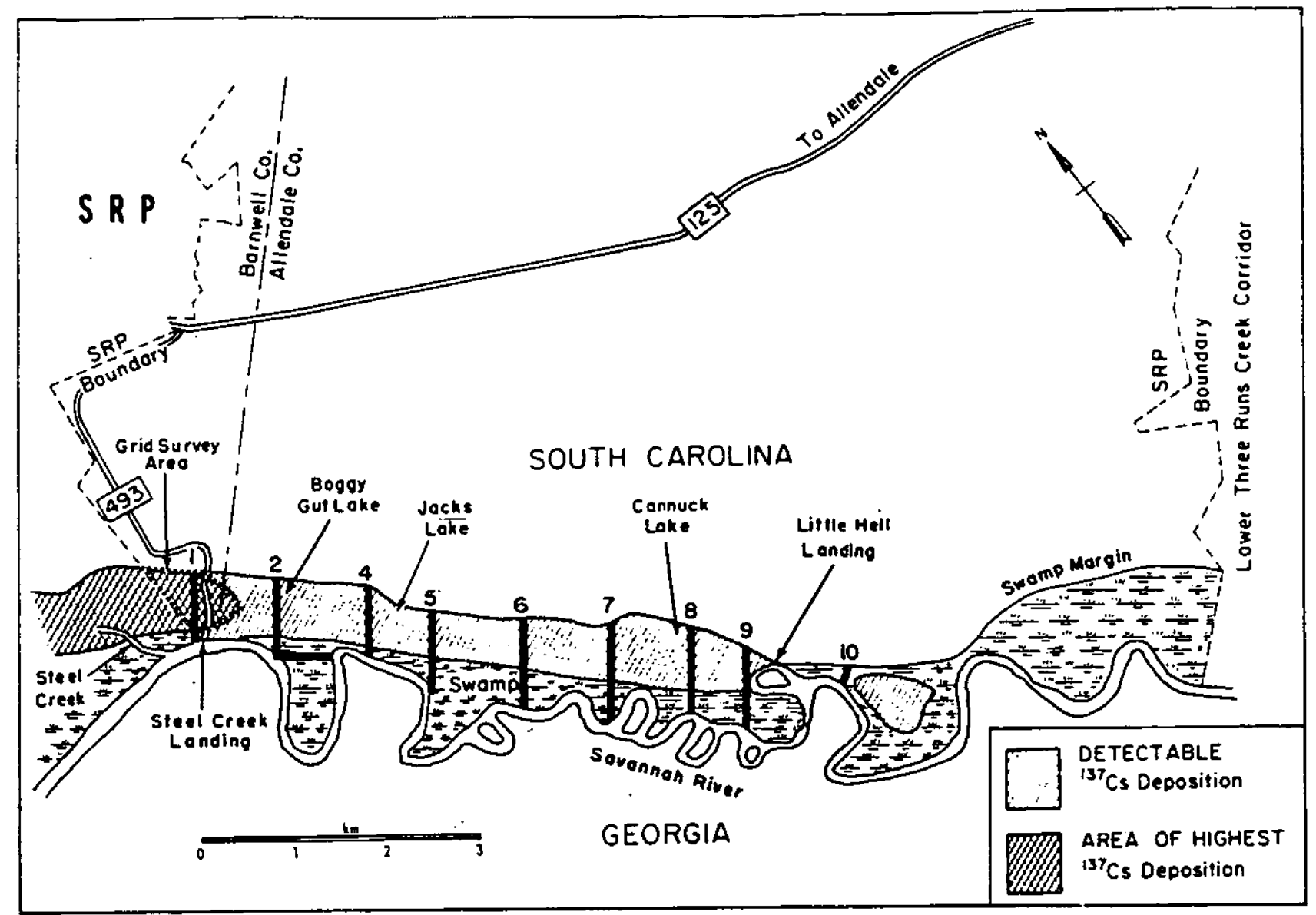

FIGURE HP.3. RADIOACTIVITY DEPOSITED IN THE SAVANNAH RIVER SWAMP 
TABLE HP-1

EISTRIBLTION CF EXPOSLRES AND ASSIMILATIONS

(IHROUCH MARCH)

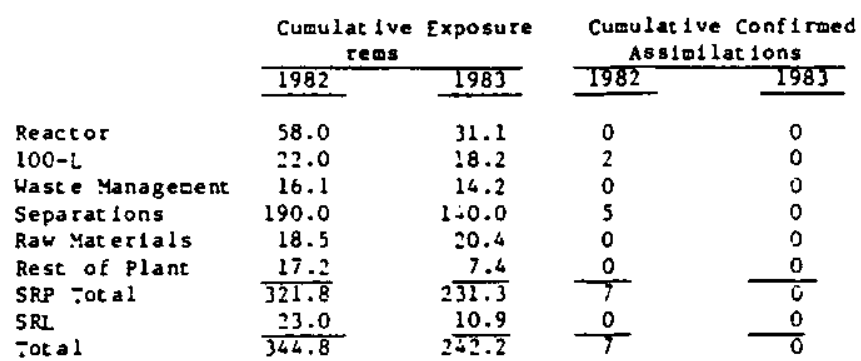

TABLE HP-2

PERSON:TL MOR:ITORINC

\begin{tabular}{|c|c|c|c|c|c|c|}
\hline & & & $\begin{array}{r}\text { Perso } \\
19 \\
\end{array}$ & $\begin{array}{l}\text { nne } 1 \\
82\end{array}$ & sure ( & $\begin{array}{l}\text { 8) } \\
33\end{array}$ \\
\hline & & & SPP & SRL & SPP & SRL \\
\hline $\begin{array}{l}\text { Mont hly Badged } \\
\text { Total Exposure } \\
\text { Whole Body Expc }\end{array}$ & $\begin{array}{l}\text { Perso } \\
\text { Year- } \\
\text { osures }\end{array}$ & $\begin{array}{l}\text { Inel-cycle } 3 \\
0-\text { Date } \\
>750 \text { mrems }\end{array}$ & $\begin{array}{l}119.2 \\
321.8 \\
11\end{array}$ & $\begin{array}{r}9.9 \\
23.0 \\
0\end{array}$ & $\begin{array}{r}89.7 \\
231.3 \\
4\end{array}$ & $\begin{array}{c}4.4 \\
10.9 \\
0\end{array}$ \\
\hline Highest Whole & body e & cposure (mren) & & & & \\
\hline & Dept. & Area & & se equ & lent & \\
\hline & WM1 & $200-F$ & & & & \\
\hline
\end{tabular}

TABLE HP-3 BIOASSAY SUMBARY

(MARCH)

\begin{tabular}{|c|c|c|c|c|c|c|}
\hline & \multirow{2}{*}{$\begin{array}{l}\text { Positive } \\
\text { Samples }\end{array}$} & \multirow{2}{*}{$\begin{array}{c}\text { Maximum } \\
\text { Concent rat 1on }\end{array}$} & \multicolumn{2}{|c|}{$\begin{array}{l}\text { Conf I roed } \\
\text { Upt akes }\end{array}$} & \multicolumn{2}{|c|}{$\begin{array}{l}\text { Number of Samples } \\
\text { Recelved }\end{array}$} \\
\hline & & & Month & $\overline{Y * D}$ & Mont h & YTD \\
\hline $\begin{array}{l}\text { Irit lum } \\
\text { Uranlum } \\
\text { Plut onfum } \\
\text { Enriched } U \text {. } \\
\text { Fission }\end{array}$ & $\begin{array}{r}934 \\
19 \\
35 \\
115\end{array}$ & $\begin{array}{l}18.0 \mu C 1 \text { HTO } / 2 \\
30 \mathrm{ug} / 1.5 \mathrm{l} \\
301 \mathrm{~d} / \mathrm{m} / 1.5 \mathrm{~L} \\
114 \mathrm{~d} / \mathrm{w} / 1.5 \mathrm{~S}\end{array}$ & $\begin{array}{l}0 \\
0 \\
0 \\
0\end{array}$ & $\begin{array}{l}0 \\
0 \\
0 \\
0\end{array}$ & $\begin{array}{r}6,344 \\
180 \\
549 \\
528\end{array}$ & $\begin{array}{r}17,644 \\
378 \\
1,365 \\
989\end{array}$ \\
\hline Aft 1 vac 1on Prod. & 0 & -- & 0 & $\mathbf{0}$ & 82 & 917 \\
\hline Americ lum-Curium & 0 & - & 0 & 0 & 65 & 113 \\
\hline Nept unlum & 0 & -- & 0 & 0 & 20 & 22 \\
\hline Lead & 0 & $\rightarrow$ & 0 & 0 & 2 & 6 \\
\hline Mercury & 0 & - & 0 & 0 & 0 & 10 \\
\hline $\begin{array}{l}1983 \text { Total: } \\
1982 \text { Totals }\end{array}$ & & . & 0 & 0 & $\begin{array}{l}7,770 \\
6,622\end{array}$ & $\begin{array}{l}\overline{21,444} \\
19,915\end{array}$ \\
\hline
\end{tabular}


TABLE HP-7

Radloactivisy in Burial Ground leget at ion, $\mathrm{PCl} / \mathrm{g}$

Sample Locarion (F1Bure EM-5)

\begin{tabular}{|c|c|}
\hline$\frac{E M-5)}{1}$ & $\frac{\text { Alpha }}{0.20+0.1}$ \\
\hline A & $0.06+.13$ \\
\hline & $0.42+.25$ \\
\hline 3 & $0.55 \pm .28$ \\
\hline 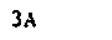 & $0.26 \pm .20$ \\
\hline 4 & $0.36 \pm .23$ \\
\hline $4 \mathrm{~A}$ & $0.20 \pm .18$ \\
\hline 5 & $0.03 \pm .11$ \\
\hline 6 & $0.29 \pm .23$ \\
\hline 7 & $0.20 \pm .18$ \\
\hline 8 & $0.20 \pm .18$ \\
\hline $8 \mathbf{A}$ & $0.23 \pm .13$ \\
\hline 9 & $0.20 \pm .18$ \\
\hline $9 A$ & $0.32 \pm .22$ \\
\hline 10 & $0.26 \pm .21$ \\
\hline 11 & $0.16 \pm .17$ \\
\hline 12 & $0.13 \pm .16$ \\
\hline 13 & $0.32 \pm .22$ \\
\hline 14 & $0.23 \pm .22$ \\
\hline $14 \mathrm{~A}$ & $0.07 \pm .08$ \\
\hline 5 & $0.36 \pm .25$ \\
\hline
\end{tabular}

$k-40$
$10 \pm 5.7$
$9 \pm 5.4$
$7 \pm 4.9$
$10 \pm 4.3$
$7 \pm 4.6$
$16 \pm 5.1$
$26 \pm 7.1$
$10 \pm 5.8$
$11 \pm 7.6$
$8 \pm 5.5$
$21 \pm 6.3$
$8 \pm 5.6$
$12 \pm 5.8$
$12 \pm 5.5$
$18 \pm 5.5$
$13 \pm 6.0$
$13 \pm 5.8$
$19 \pm 8.8$
$8 \pm 5.5$
$13 \pm 7.3$
$19 \pm 8.0$

(1)

Sample Loc ation (E1gure EM-5)

$$
16
$$

17

18

19

$19 \lambda$

20

$20 \mathrm{~A}$

21

22

23

$23 \mathrm{~A}$

24

25

26

27

28

29

30

31

32

Alpha
$0.12 \pm .10$
$0.06 \pm .13$
$0.03 \pm .12$
$0.20 \pm .18$
$0.16 \pm .17$
$0.10 \pm .09$
$0.10 \pm .14$
$0.42 \pm .25$
$0.03 \pm .12$
$0.96 \pm .37$
$0.16 \pm .17$
$0.22 \pm .20$
$0.32 \pm .22$
$0.00 \pm .09$
$0.36 \pm .24$
$0.16 \pm .19$
$0.03 \pm .12$
$0.06 \pm .13$
$0.16 \pm .17$
$0.03 \pm .12$
$0.03 \pm$

$\begin{array}{ll}\frac{1-20}{13 \pm 5.5} & \frac{5.5-137}{58 \pm 0.55} \\ 10 \pm 5.6 & 1.1 \pm 0.56 \\ 4 \pm 15 & 17 \pm 1.2 \\ 2 \pm 6.3 & 5.08 \pm 1.5 \\ 9 \pm 6.3 & 0.16 \pm 0.59 \\ 9 \pm 6.3 & 0.31 \pm 0.52 \\ 2 \pm 5.3 & 0 \pm 0.53 \\ 17 \pm 5.5 & 0.31 \pm 0.50 \\ 23 \pm 0.6 & 0.57 \pm 0.52 \\ 8 \pm 5.2 & 0.50 \pm 0.53 \\ 8 \pm 5.8 & 3.4 \pm 5.5 \\ 8.3 \pm 5.2 & 2.6 \pm 5.5 \\ 5 \pm 5.4 & 1.8 \pm 0.69 \\ 4 \pm 5.6 & 1.1 \pm 0.65 \\ 11 \pm 5.7 & 3 \pm 0.34 \\ 8 \pm 5.6 & 0.19 \pm 5.5 \\ 15 \pm 6.7 & 0.64 \pm 0.59 \\ 9 \pm 5.6 & 0.68 \pm 0.53 \\ 20 \pm 6.4 & 1.2 \pm 0.61 \\ 7 \pm 5.0 & 1.0 \pm 0.52 \\ 15 & \end{array}$

REFERENCE:

25-M1le Rad1us Veget at Ion - 1982

Maximum Concentrat $10 \mathrm{n}, \mathrm{pCl} / \mathrm{s}$

Alpha $\frac{\text { Maxiaum Concent }}{0.92+0.38}$

$\begin{array}{ll}\mathrm{K}-40 & 8.3+-6.6 \\ \mathrm{C}=-134,137 & 1.1+0.9\end{array}$ 


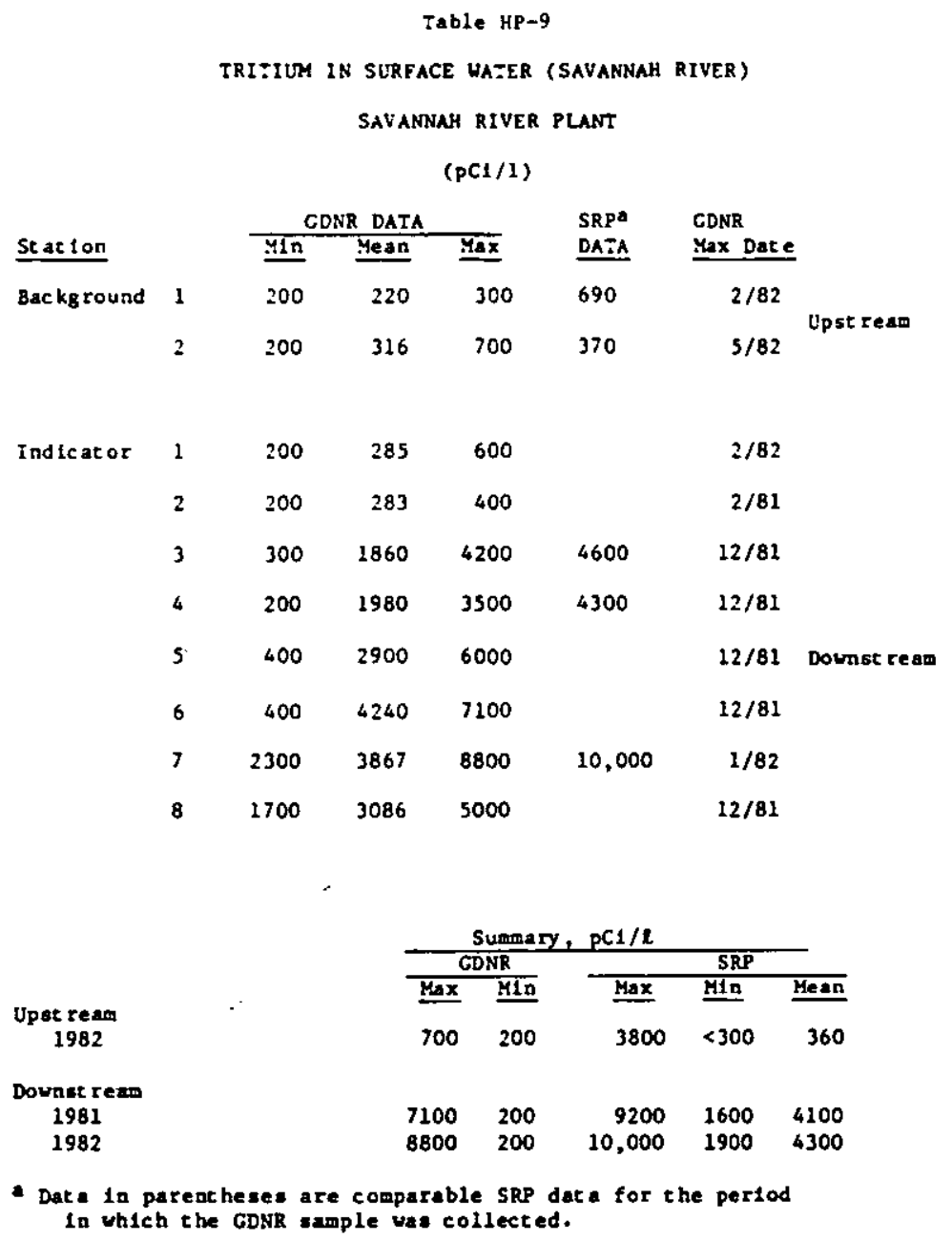




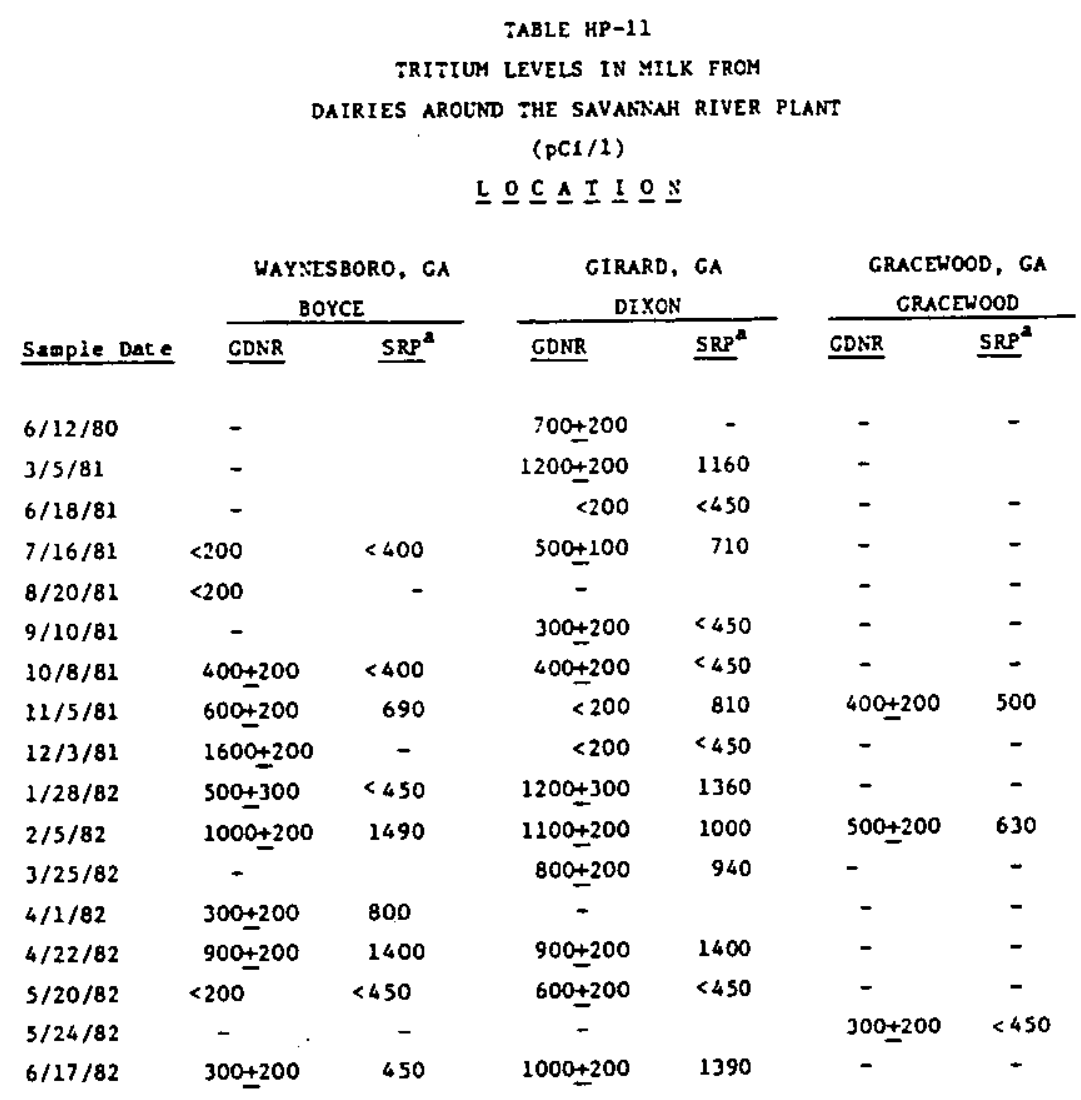

a Comparable SRP data. 


\begin{tabular}{|c|c|c|c|c|c|}
\hline \multirow[b]{2}{*}{$\begin{array}{l}\text { River } \\
\text { Mile }\end{array}$} & \multirow[b]{2}{*}{$\begin{array}{l}\text { Trail } \\
\text { Number }\end{array}$} & \multicolumn{3}{|c|}{$\begin{array}{c}\text { TABLE HP-13 } \\
\text { SAVANAH RIVER SWAMP } \\
\text { STEEL CREEK TO LITTLE HELL LNNDING } \\
\text { TLD RADIATION MEASUREMENTS } \\
\end{array}$} & \multirow[b]{2}{*}{$\begin{array}{l}\text { Oct.b } \\
1982\end{array}$} \\
\hline & & $\begin{array}{l}\text { Distance } \\
\text { Frow } \\
\text { River } \\
\text { (Mecers) } \\
\end{array}$ & $\begin{array}{l}\frac{m R / D a y}{\text { Average Annual a }} \\
\text { Resulte } \\
1972-1980 \\
\end{array}$ & $\begin{array}{c}\text { Sept. } \\
1981 \\
\end{array}$ & \\
\hline 141.5 & 1 & 0 & $0.27 \pm 0.03$ & $.0 .20 \pm 0.02$ & $0.23 \pm 0.02$ \\
\hline & & $\begin{array}{l}178 \\
358 \\
550\end{array}$ & $\begin{array}{l}0.34 \pm 0.07 \\
0.52 \pm 0.09 \\
1.11 \pm 0.21\end{array}$ & $\begin{array}{l}0.35=0.03 \\
0.50 \pm 0.04 \\
1.13 \geq 0.08\end{array}$ & $\begin{array}{l}0.34=0.03 \\
0.50 \div 0.04 \\
1.02 \pm 0.07\end{array}$ \\
\hline & & $\begin{array}{l}656 \\
805\end{array}$ & $\begin{array}{l}1.46 \pm 0.25 \\
0.17 \pm 0.03\end{array}$ & $\begin{array}{l}1.08=0.08 \\
0.17=0.02\end{array}$ & $\begin{array}{l}1.02 \pm 0.08 \\
0.14 \pm 0.01\end{array}$ \\
\hline 140.8 & 2 & $\begin{array}{r}0 \\
207 \\
406 \\
598 \\
798 \\
945 \\
975\end{array}$ & $\begin{array}{l}0.21 \pm 0.03 \\
0.25 \pm 0.03 \\
0.24 \pm 0.03 \\
0.25 \pm 0.02 \\
0.33 \pm 0.04 \\
0.59 \pm 0.04 \\
0.18 \pm 0.02\end{array}$ & $\begin{array}{l}0.24: 0.02 \\
0.23: 0.02 \\
0.26: 0.02 \\
0.23: 0.02 \\
0.35 \div 0.03 \\
0.50 \pm 0.04 \\
0.16 \pm 0.02\end{array}$ & $\begin{array}{l}0.23 \pm 0.02 \\
0.28 \pm 0.03 \\
0.27 \pm 0.02 \\
0.29 \pm 0.03 \\
0.38 \pm 0.03 \\
0.51 \pm 0.04 \\
0.19 \pm 0.02\end{array}$ \\
\hline 139.5 & 3 & 0 & $0.23 \pm 0.03$ & $0.22 \div 0.02$ & $0.23 \pm 0.02$ \\
\hline $\begin{array}{l}\text { to } \\
140.8\end{array}$ & & $\begin{array}{l}281 \\
627\end{array}$ & $\begin{array}{l}0.25 \pm 0.06 \\
0.24 \pm 0.01\end{array}$ & $\begin{array}{l}0.25 \geq 0.02 \\
0.21 \pm 0.02\end{array}$ & $\begin{array}{l}0.28 \pm 0.03 \\
0.23 \pm 0.02\end{array}$ \\
\hline 139 & 4 & $\begin{array}{r}0 \\
293 \\
380 \\
515 \\
580 \\
729\end{array}$ & $\begin{array}{l}0.28 \pm 0.02 \\
0.29 \pm 0.04 \\
0.39 \pm 0.07 \\
0.39 \pm 0.08 \\
0.82 \pm 0.10 \\
0.30 \pm 0.19\end{array}$ & $\begin{array}{l}0.26: 0.02 \\
0.35: 0.03 \\
0.39: 0.03 \\
0.43: 0.03 \\
0.82: 0.06 \\
0.25 \pm 0.02\end{array}$ & $\begin{array}{l}0.24 \pm 0.02 \\
0.34 \pm 0.03 \\
0.42 \pm 0.03 \\
0.43 \pm 0.03 \\
0.80 \pm 0.06 \\
0.25 \pm 0.02\end{array}$ \\
\hline 138.5 & 5 & $\begin{array}{r}0 \\
534 \\
573 \\
640 \\
773\end{array}$ & $\begin{array}{l}0.23 \pm 0.04 \\
0.34 \pm 0.04 \\
0.58 \pm 0.05 \\
1.05 \pm 0.14 \\
0.25 \pm 0.03\end{array}$ & $\begin{array}{l}0.26 \pm 0.02 \\
0.36 \pm 0.03 \\
0.56 \pm 0.04 \\
1.03 \pm 0.07 \\
0.24 \pm 0.02\end{array}$ & $\begin{array}{l}0.26 \pm 0.02 \\
0.35 \pm 0.03 \\
0.55 \pm 0.04 \\
1.00 \pm 0.07 \\
0.28 \pm 0.03\end{array}$ \\
\hline
\end{tabular}

- No Analyais

a The average volues are accompanied by a plus or minus $(t)$ limit value, which is the 2 sigma standard deviation of the average.

b The individual 1981 and 1982 resulto are accompanied by a \pm value which represents che statiatical counting error at the 952 confidence level. 
TABLE HP-14

SAVANNAH RIVER SHAMP -- STEEL CREEK TO LITTLE HELL LANDING RACIOACTIVITY IA SOIL

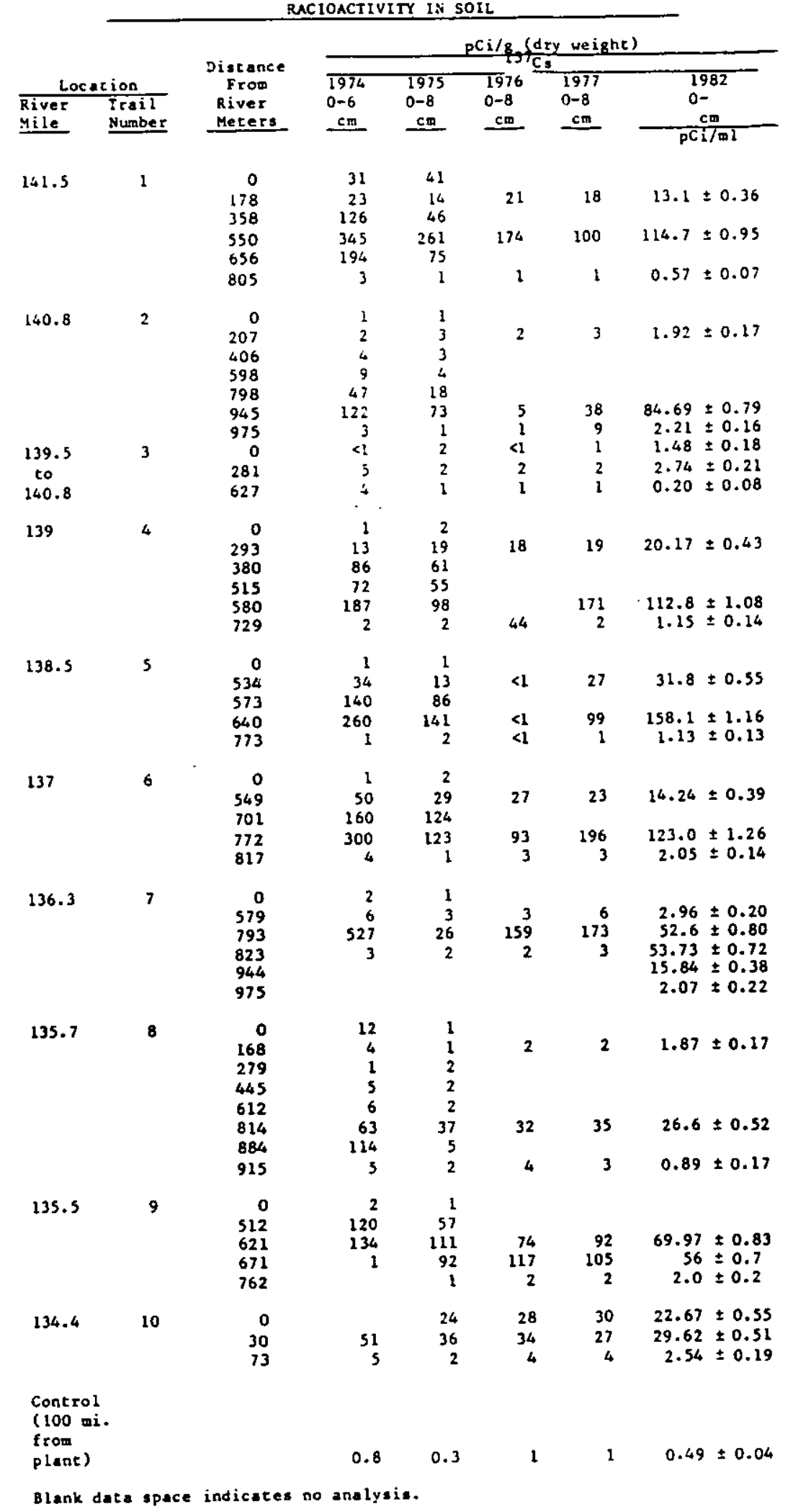


TABLE HP-16

RADIOACTIVITY IN AQUATIC SPECIES FROH

LAKES IN SAVAMRAH RIVER SWMMP, a $\mathrm{PCi} / \mathrm{g}$ (wet wt)

\begin{tabular}{|c|c|c|c|c|c|}
\hline & Species & Number & & & $88^{\circ}$ \\
\hline & or type & of Fish & Max & $A v g$ & \\
\hline $\begin{array}{c}\text { Trail } \\
\text { Boggy Gut }\end{array}$ & & & & & \\
\hline 1974 & Compositeb & 7 & 6.1 & 3.8 & \\
\hline 1975 & Bess: & 6 & 4.5 & 2.6 & \\
\hline & Bream & 2 & 1.7 & 1.4 & $6^{b}$ \\
\hline 1977 & Catfish & 2 & 0.2 & 0.2 & $<5$ \\
\hline & Bream & 3 & 0.2 & 0.1 & $\ll$ \\
\hline 1982 & Breat & 5 & $0.9 \pm 1.1$ & $0.4 \pm 0.6$ & \\
\hline $\begin{array}{r}\text { Trail } 7 \\
\text { Jack Lak }\end{array}$ & & & & & \\
\hline 1974 & Composite & 7 & 5.8 & 4.0 & \\
\hline 1975 & Bas: & 1 & 4.5 & 4.5 & $4^{b}$ \\
\hline & Bream & 2 & 2.1 & 1.3 & \\
\hline 1977 & Breas & 1 & $\infty .1$ & $\infty .1$ & $\omega$ \\
\hline & Carp & $i$ & $<0.1$ & $<0.1$ & $\ll$ \\
\hline & Crappie & i & $\infty .6$ & $\infty .6$ & 8 \\
\hline 1982 & Bass & 1 & $0.5 \pm 0.3$ & $0.5 \pm 0.3$ & \\
\hline & Bream & 5 & $0.7 \pm 1.1$ & $0.4 \pm 0.3$ & \\
\hline & Jack & 2 & $0.5 \pm 0.1$ & $0.4 \pm 0.1$ & \\
\hline & Sucker & 2 & $0.2 \neq 0.2$ & $0.2 \pm 0.1$ & \\
\hline & Crappie & 1 & $0.3 \pm 0.4$ & $0.3 \pm 0.4$ & \\
\hline & Turtie & 1 & $0.2 \pm 0.2$ & $0.2 \pm 0.2$ & \\
\hline
\end{tabular}

Trail 8

Cennuck Lake

$\begin{array}{ll}1974 & \text { Compositeb } \\ 1975 & \text { Bream } \\ 1977 & \text { Catfinh } \\ 1982 & \text { Bream } \\ & \text { Jack } \\ & \text { Sucker } \\ & \text { Creppie } \\ & \text { Turtle }\end{array}$

Control

Qiver 2

$\begin{array}{llrlll}1974 & \text { All speciesc } & 89 & 1.8 & 1.1 & 5 \\ 1975 & \text { All speciesc } & 41 & 2.4 & 0.1 & 5 \\ 1976 & \text { Catfish } & 6 & 0.1 & 0.1 & 0 \\ 1977 & \text { Brean } & 8 & 0.1 & 0.1 & 3 \\ 1982 & \text { Catfish } & 9 & 0.1 & \$ .1 & 2\end{array}$

a No fith collected in 1976.

Composite of all species.

cCatfish, bream, and bast.

B lank data space iodicate: no analyois

5

$0.3 \pm 0.3$

$0.1 \pm 0.3$

$0.6 \pm 0.4$

$0.3 \pm 0.4$
$0.4 \pm 0.03$

$0.6 \pm 0.8$

$0.1 \pm 0.3$

$0.5+0.2$

$$
\begin{aligned}
& 5 \\
& 9 \\
& \\
& 5 \\
& 5 \\
& 3 \\
& 2
\end{aligned}
$$


TABLE HP-19

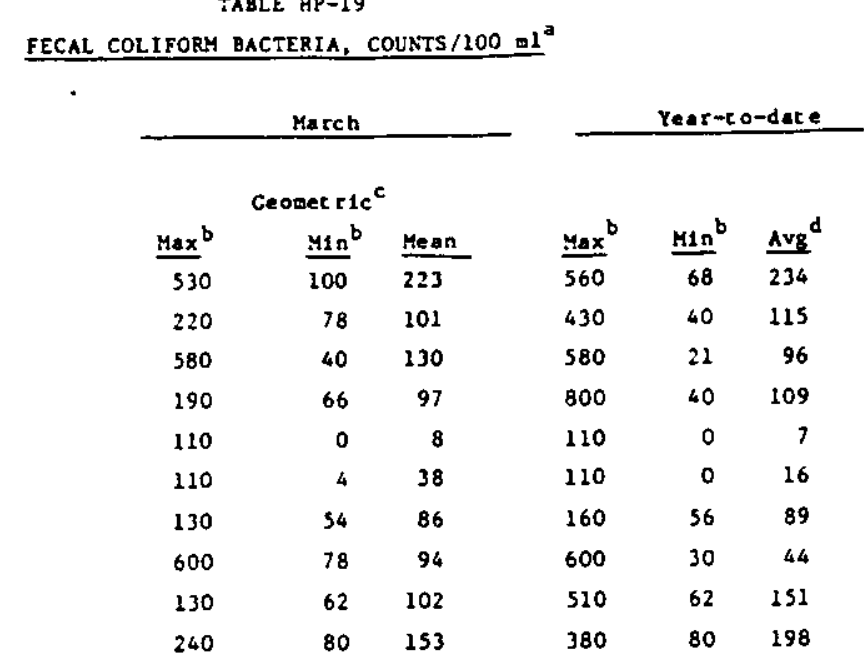

R1ver 2, above plant

R1ver 10, below plane

Upper Three Runs at Road $f$

Upper Three Runs at Road A

Four Mlle at Road $A$

Pen Branch at Road $A$

Steel Creek at Road $A$

Beaver Dam Creek near swamp

Lower Three Runs at Road A

Lower Three Runs at

Iabernacle Church Road

- Weekly analyses. Locar ion of sample polncs are hown in Appendix $A$.

Figure HP-6.

b Maximum and olnimum values for veekly analyses.

c Geometric mean of weekly values. The standard for South carolina staces chat the fecal collfort count should: Not exceed a geomer rle mean of $1000 / 100$ l based on five consecut ive samples during any 30 day period; nor to exceed 2000/100ml in wore than $20 \%$ of the samples exafined during such period (not applicable during or follouiag pariode of rainfa11).

d Average of wothly geotet ric meana. 


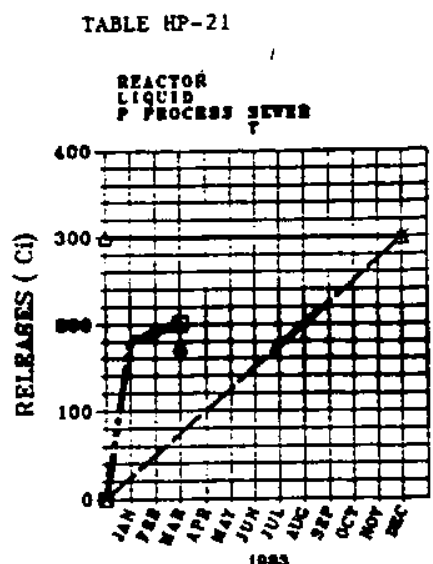

10as

PROBABLE CAUSE: RESULIED FROM PLNPING LIQUID

FROM THE DISTILLATION PAD HOLDING TANR IN JANUARY.

MARG RELEASES WERE NORMAL.

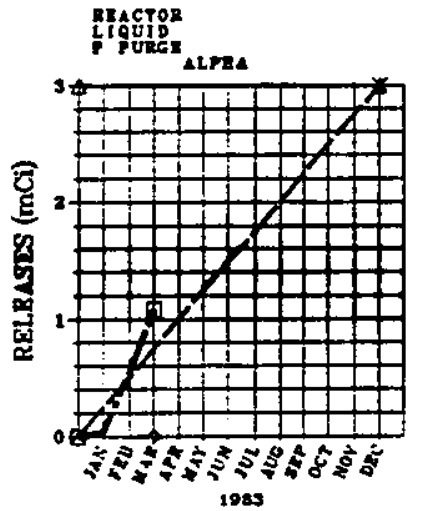

1985

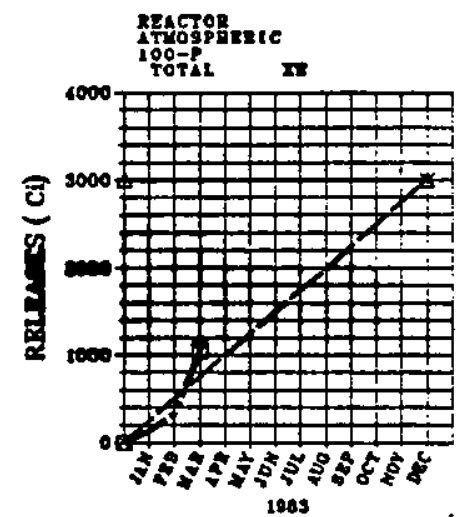

1905

PROBABLE CAUSE: PROCEDURAL PURGE REQUIRED TO REDUCE TRITIUY CONCENTRATIONS.

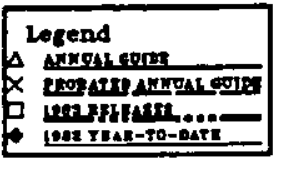

E topa

ens Y TAE-70-0atz

PROBABLE CAUSE: MARK 31-A TARGET FAILURE $3 / 2 / 83$. 

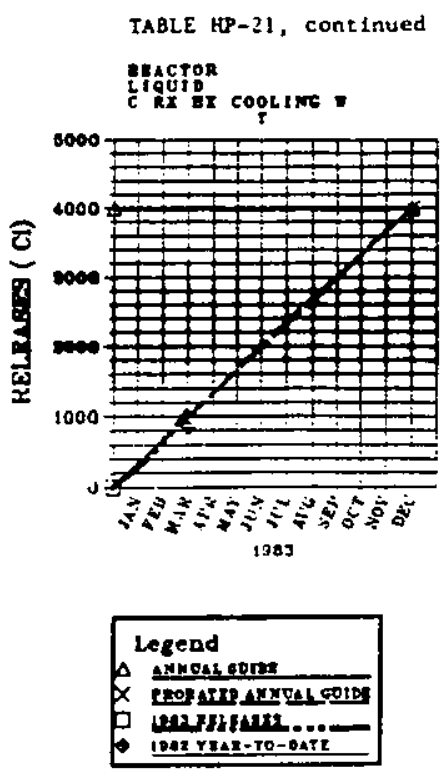

PROBABLE CAUSE: SEVERAL SMALI RELEASES DURING MARCH.
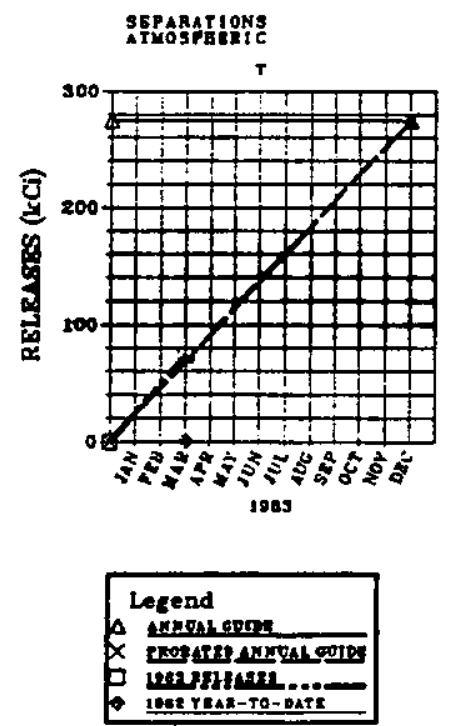

PROBABLE CAUSE: NORMAL OPERATIONS THAT CAUSED RELEASES TO REMAIN CLOSE TO THE GUIDE. 
TABLE HP-22

BEACIOR AREA RADTOACIUVERELEASES

MARCH 1983

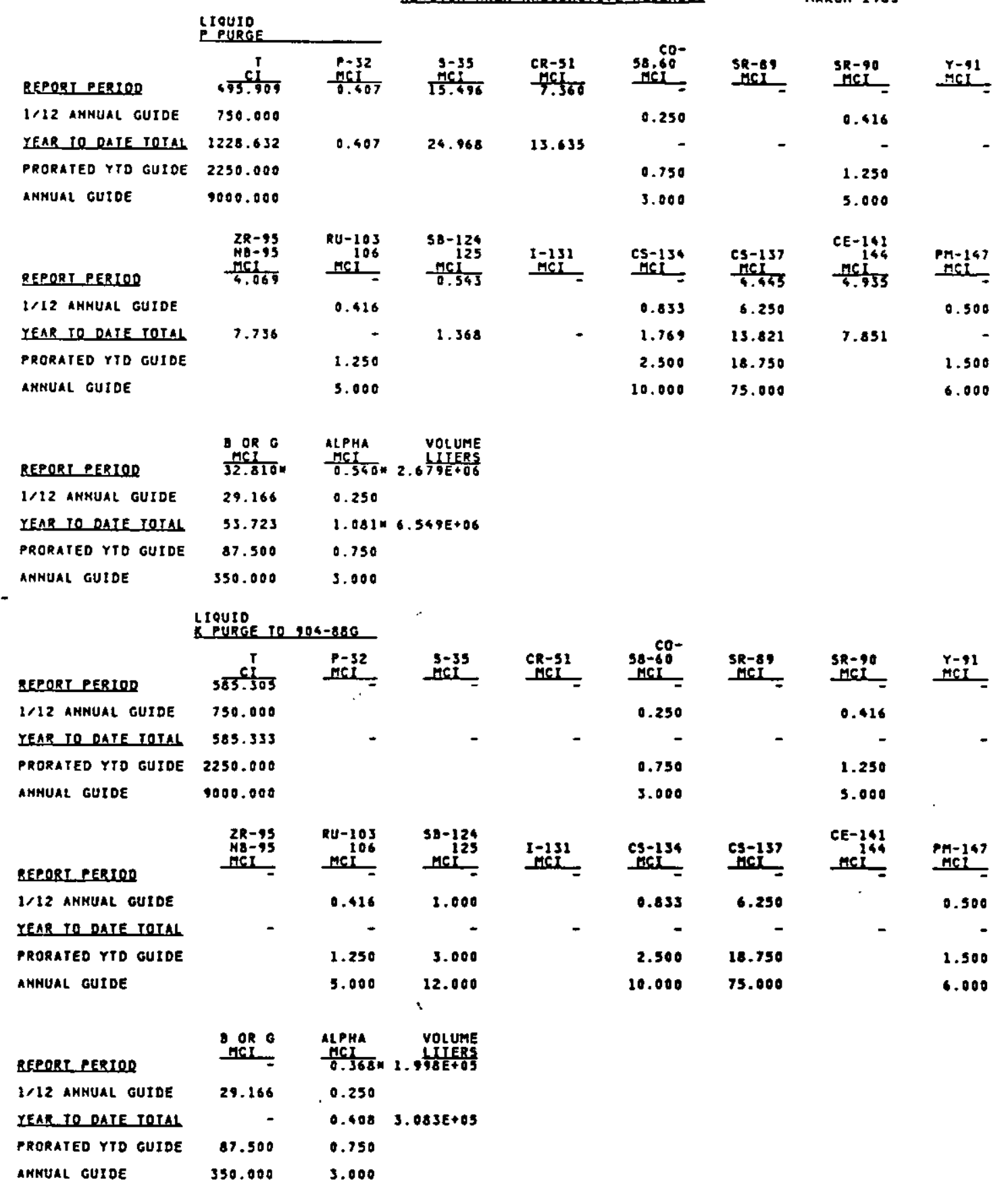

= ACCURACY MOT ALWAYS IMPLIED BY MUMDER OF SIGNIFICANT DIGITS EXXEEDS PERIOD ANDJOR YTD GUIDES

- LESS THAH SENSITIVITY OF ANALYSIS

MCI $=$ MILLICURIES

1 


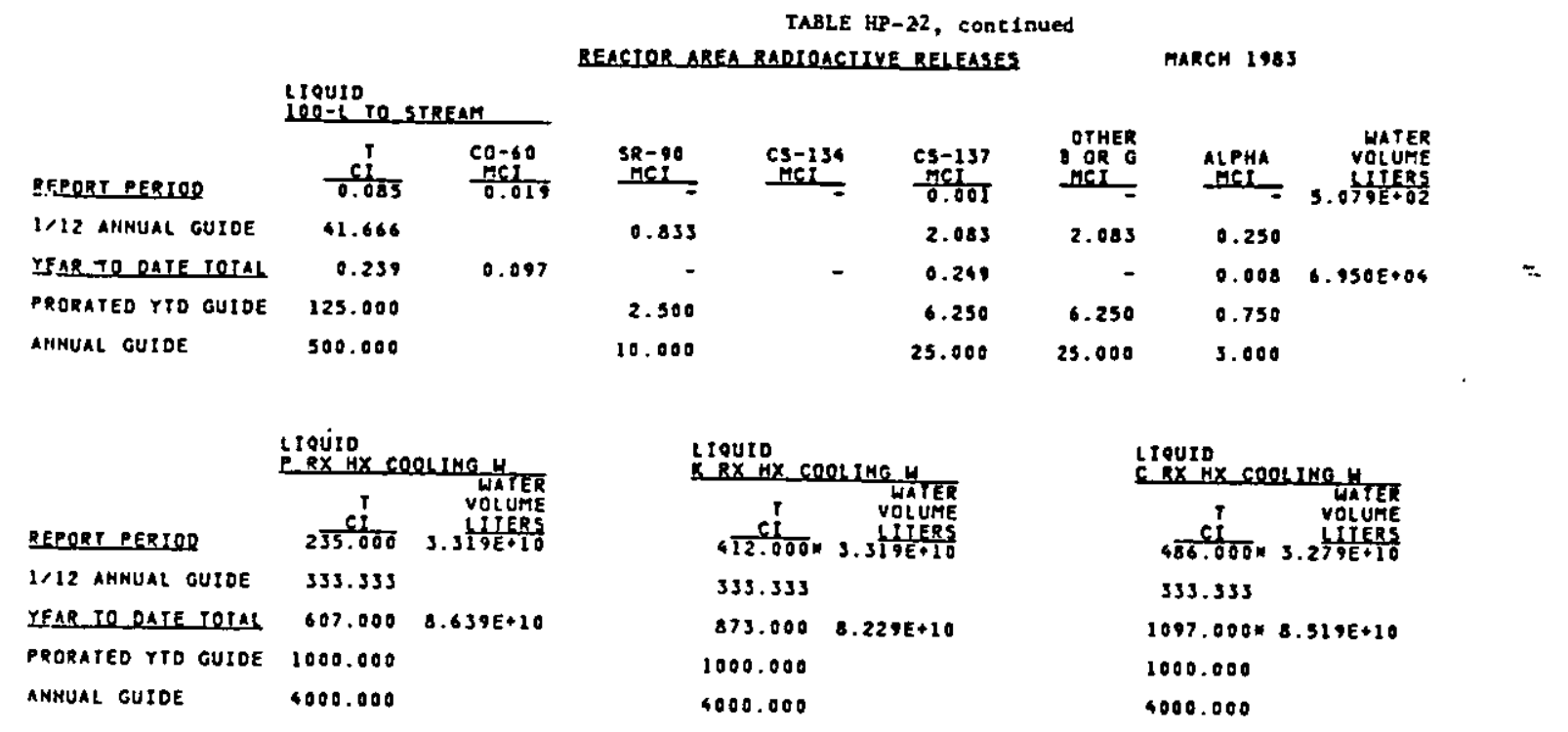

\begin{tabular}{|c|c|c|c|c|c|c|c|}
\hline \multirow[b]{2}{*}{ RFPORT EERIOD } & \multicolumn{2}{|c|}{$\begin{array}{l}\text { LQUYD } \\
690-6 \text { SEEPAGE_BASIN }\end{array}$} & \multicolumn{2}{|l|}{. } & \multirow{2}{*}{$\begin{array}{r}\text { OTKER } \\
\text { OTR } \\
\text { OREL } \\
\end{array}$} & \multirow[b]{2}{*}{ - } & \multirow[b]{2}{*}{$\begin{array}{r}\text { VOLUTE } \\
0.0 \frac{\text { UTERS }}{0.05+0 S}\end{array}$} \\
\hline & $\stackrel{T}{C_{1}}$ & $\begin{array}{l}{ }_{\text {MCI }}^{C O-60} \\
\end{array}$ & $\begin{array}{l}\text { SR-90 } \\
\text { MCI }\end{array}$ & $\begin{array}{l}\text { CS-137 } \\
\text { MEI }\end{array}$ & & & \\
\hline I/12 ANNUAL GUIDE & 8.333 & $0.083^{\circ}$ & 0.083 & 0.083 & 0.166 & 0.083 & \\
\hline YEAB TO DATE TOTAS & 0.005 & 0.018 & - & - & - & 0.001 & $2.269 E+04$ \\
\hline PRORATEO YTD GUIDE & 25.000 & 0.250 & 0.250 & 0.250 & 0.500 & 0.230 & \\
\hline ammUAC GUIDE & 100.000 & 1.000 & 1.000 & 1.000 & 2.000 & 1.000 & \\
\hline
\end{tabular}

- ACCURACY NOT ALWAYS IMPLIED BY RUMBER OF SIGNIFICANT DIGITS " EXCEEDS PERIOD ANDJOR YTO GUIDES

BCI $=$ MILLSTCURIES

I 
TABLE HP-23, continued

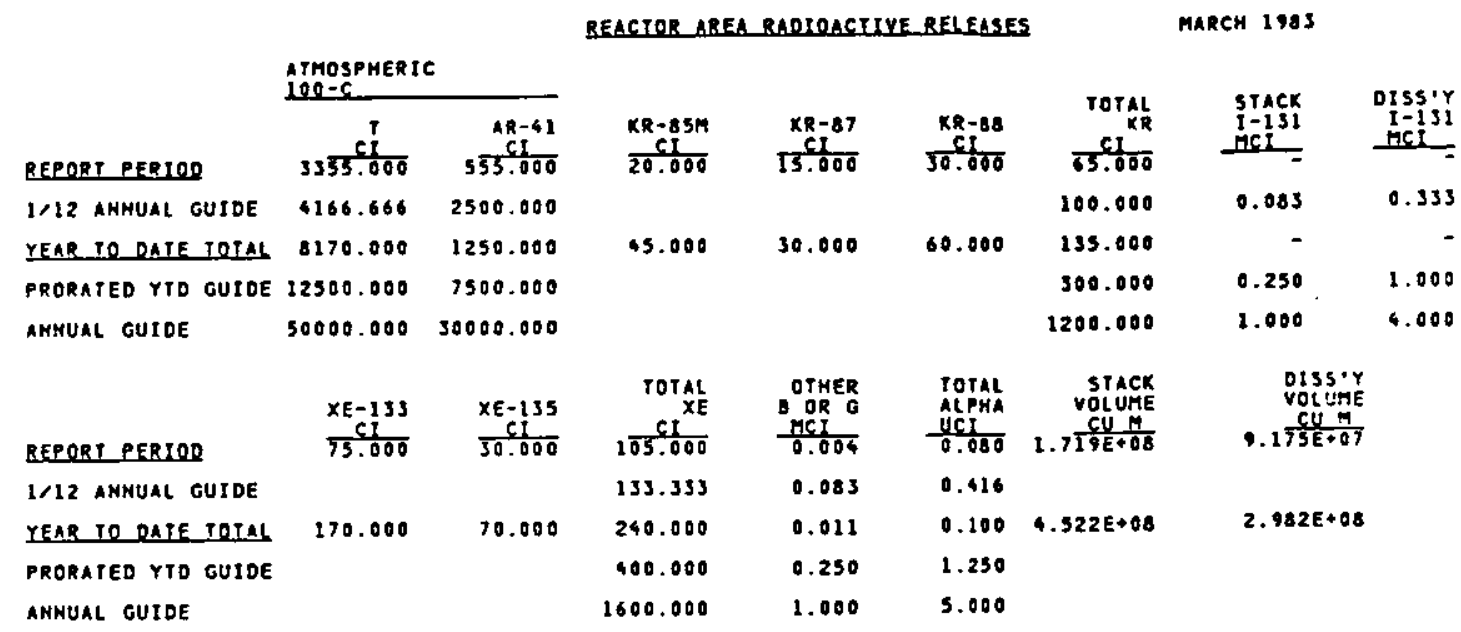

- ACCURACY NOT ALWAYS IMPlIED BY NUMBER OF SIGHIFICANT DIOITS W EXCEEDS PERIOD ANDIOR YTO GUIDES MESS THAN SENSITIVITY OF AHALYSIS 
TABLE HP-25

SEPARATIOHS AREA BADTOACIIYE RELEASES

MARCH 1983

HeUIR to STREAMS

\begin{tabular}{|c|c|c|c|c|c|c|}
\hline 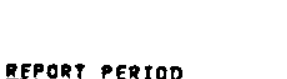 & ci & $\begin{array}{l}5 R-89 \\
M C T^{96} \\
\end{array}$ & $\begin{array}{c}\operatorname{cs}-136 \\
137 \\
M C 1\end{array}$ & $\begin{array}{r}\text { OTKER } \\
\text { goR G } \\
\text { HEt } \\
\end{array}$ & $\begin{array}{l}\text { Total } \\
\text { Alpha } \\
\text { Her. }\end{array}$ & $\begin{array}{l}\text { WATER } \\
\text { VOLUME } \\
\text { LUERS }\end{array}$ \\
\hline $\begin{array}{l}200-F \text { EFFLUENT } \\
200-H \text { EFFLUENT }\end{array}$ & $\begin{array}{l}1.030 \\
6.130\end{array}$ & $\begin{array}{l}0.270 \\
0.520\end{array}$ & 1.920 & 1.660 & 0.240 & $\begin{array}{l}2.7 E+08 \\
8.7 E+07\end{array}$ \\
\hline$\frac{\text { PERTOD TOTAS }}{\text { II ANAUAL GUIDE }}$ & $\begin{array}{r}7.160 \\
12.500\end{array}$ & $\begin{array}{l}0.790 \\
2.916\end{array}$ & $\begin{array}{l}2.960 \\
6.250\end{array}$ & $\begin{array}{r}2.150 \\
14.583\end{array}$ & 0.330 & $3.6 E+08$ \\
\hline $\begin{array}{l}\text { YEAR TO DATE } \\
200-F \text { EFFTUENT } \\
200-H \text { EFFLUENT }\end{array}$ & $\begin{array}{r}3.160 \\
14.820\end{array}$ & $\begin{array}{l}1.290 \\
1.230\end{array}$ & $\begin{array}{l}5.420 \\
3.910\end{array}$ & $\begin{array}{r}5.590 \\
12.010\end{array}$ & $\begin{array}{l}0.680 \\
0.210\end{array}$ & $\begin{array}{l}9.2 E+08 \\
1.7 E+08\end{array}$ \\
\hline$\frac{\text { YTD TOTAL }}{\text { PRORATED YTD GUIDE }}$ & $\begin{array}{l}17.980 \\
37.500\end{array}$ & $\begin{array}{l}2.520 \\
8.748\end{array}$ & $\begin{array}{r}9.350 \\
18.750\end{array}$ & $\begin{array}{l}17.600 \\
43.749\end{array}$ & 0.890 & $1.1 E+09$ \\
\hline AHNUAL CUIDE & 150.000 & 35.000 & 75.000 & 175.000 & 10.000 & \\
\hline
\end{tabular}

TABLE MP-26

SEPARAIIOHS AREA RADIOACTIUE RELEASES MARCH 1983

ATMOSPHERIC RELEASES

\begin{tabular}{|c|c|c|c|c|c|c|c|c|}
\hline xeí & $\begin{array}{c}5 R-89 \\
90 \\
C I\end{array}$ & $\underset{M C I}{2 R-95}$ & $\begin{array}{l}\text { NA }-95 \\
\text { MCI }\end{array}$ & $\begin{array}{c}R U-103 \\
\text { Mef }\end{array}$ & $\begin{array}{l}R U-106 \\
\mathrm{MCl}\end{array}$ & $\begin{array}{l}I-131 \\
\text { IfI }\end{array}$ & $\begin{array}{l}\operatorname{cs-134} \\
\text { ret }\end{array}$ & 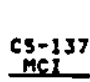 \\
\hline $24.81 \overline{ }$ & 0.211 & $\begin{array}{l}0.667 \\
0.121\end{array}$ & $\begin{array}{l}1.714 \\
0.220\end{array}$ & 0.219 & $\begin{array}{l}0.637 \\
1.327\end{array}$ & 3.622 & 0.002 & $\begin{array}{l}0.073 \\
0.016\end{array}$ \\
\hline $\begin{array}{l}24.819 \% \\
22.916\end{array}$ & 0.220. & $\begin{array}{l}0.788 \\
2.083\end{array}$ & $\begin{array}{l}1.234 \\
6.256\end{array}$ & $\begin{array}{l}0.353 \\
8.333\end{array}$ & 25.000 & 20.835 & 0.002 & $\begin{array}{l}0.089 \\
0.250\end{array}$ \\
\hline
\end{tabular}

IJíz AIBUAL GUIDE
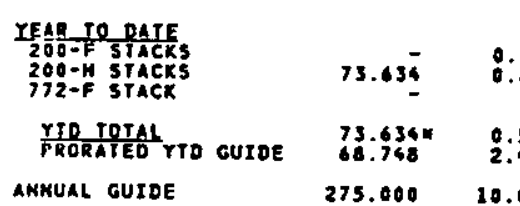

\begin{tabular}{|c|c|c|c|c|c|c|c|c|c|}
\hline 73.634 & $\begin{array}{r}0.503 \\
0.009 \\
-\end{array}$ & $\begin{array}{r}1.692 \\
0.563\end{array}$ & $\begin{array}{r}3.836 \\
0.749\end{array}$ & $\begin{array}{r}0.521 \\
0.352\end{array}$ & $\begin{array}{r}1.637 \\
2.547\end{array}$ & $\begin{array}{r}4.679 \\
0.693 \\
-\end{array}$ & $0.00 \bar{s}$ & $\begin{array}{r}0.172 \\
0.056\end{array}$ & \\
\hline $\begin{array}{l}73.6344 \\
68.748\end{array}$ & $\begin{array}{l}0.512 \\
2.496\end{array}$ & $\begin{array}{l}2.255 \\
6.249\end{array}$ & $\begin{array}{r}4.585 \\
18.750\end{array}$ & $\begin{array}{r}0.873 \\
24.994\end{array}$ & 73.184 & $\begin{array}{r}5.374 \\
62.499\end{array}$ & 0.003 & $\begin{array}{l}0.228 \\
0.750\end{array}$ & \\
\hline 275.000 & 10.000 & 25.000 & 75.000 & 100.000 & 300.000 & 250.000 & .500 & 3.000 & \\
\hline $\begin{array}{l}C E-161 \\
M C I\end{array}$ & $\begin{array}{l}C E-144 \\
\text { ECI }\end{array}$ & 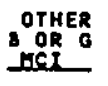 & $\begin{array}{r}\operatorname{Ar}-241 \\
243 \\
-\operatorname{arct}\end{array}$ & $\begin{array}{r}c 91-242 \\
244 \\
\end{array}$ & $\begin{array}{r}u-235 \\
238 \\
-101\end{array}$ & $\begin{array}{r}P U-238 \\
239 \\
-18\end{array}$ & $\begin{array}{l}\mathrm{Pu}-238 \\
\mathrm{MCt}\end{array}$ & $\begin{array}{l}\text { P-239 } \\
\text { ner }\end{array}$ & $\begin{array}{l}\text { AIR } \\
\text { YOLUME } \\
\text { CYLM }\end{array}$ \\
\hline 0.036 & $\begin{array}{l}0.844 \\
0.191\end{array}$ & $=$ & 0.011 & 0.016 & 0.269 & 0.055 & $\begin{array}{l}0.023 \\
0.122\end{array}$ & 0.032 & $\begin{array}{l}3.4 E+\theta 8 \\
2.2 E+08\end{array}$ \\
\hline $\begin{array}{l}0.036 \\
0.166\end{array}$ & $\begin{array}{l}1.035 \\
2.500\end{array}$ & $5: 00 \overrightarrow{0}$ & $\begin{array}{l}0.014 \\
0.166\end{array}$ & $\begin{array}{l}0.025 \\
0.166\end{array}$ & $\begin{array}{l}0.282 \\
0.033\end{array}$ & $\begin{array}{c}0.189 \\
0.835\end{array}$ & 0.145 & 0.044 & $5.7 E+68$ \\
\hline
\end{tabular}

YEARTO DATE
$200-F$ STACKS
$200-H$ STACKS
$772-F$ STACK
YTO TOTAL
PRORATED YTD GUIDE
AHNUAL GUIDE

$\begin{array}{rrr}0.091 & 2.038 & 0.005 \\ 0.031 & 0.667 & 5 \\ 0.122 & 2.705 & 0.005 \\ 0.498 & 7.500 & 15.000 \\ 2.000 & 30.000 & 60.000\end{array}$

0.048
0.017
0.065
0.498
2.000

0.04

0.064

2.908

0.375

0.180

0.193

$1.15+09$

$\begin{array}{ll}1.971 & 0.861 \\ 2.499 & 2.499\end{array}$

0.56s

0.293

O. 0 E +01

$2.000 \quad 20.000 \quad 20.000$

- ACCURACY NOT ALHAYS IMPLIED BY MUMBER OF SIGNIFICANT DIGITS - EXCEEDS PERIOD AND

- LESS THAN SEHSITIUITY OF ANALYSIS

! 
TABLE HP-29

IECHNICAL AREA RADTOACIUVE RELEASES

I Q QUID

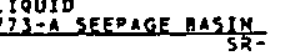

QEPQR I PERTOON

$1 / 12$ ANHUAL GUIDE

YEAR IO DATE TOTAL

PRORATED YTD GUIDE

AMHUAL GUIDE

REPORT PERINO

I/12 ANMUAL GUIDE

YEAR TO DATE TOTAL

PRORATED YTD GUIDE

AHNUAL GUIDE

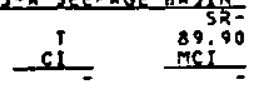

0.166

Cs- 134

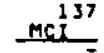

OTHER

OT 6
MEI

0.250

0.250

$0.500 \quad 0.125$

2.000

0.500

0.750

3.000

0.750

TMOSPHERIC

OTHER WATER
ALPHA VOLUME

$\stackrel{\text { MCI }}{=} 0.0 \frac{\text { UTERS }}{00 E+0 S}$

0.166

$-0.000 E+03$

213-A

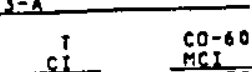

25.000

$\frac{\text { MCI }}{0.028}$

0.125

0.500

2.000

75.000

0.048

0.375

1.500

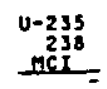

U/PU
MCI

0.250

0.750

3.000

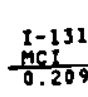

0.833

0.520

2.500

10.000

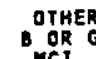

0.001
MEI G

0.025

0.001

0.075

0.300
MARCH 1983
$\begin{array}{ll}P U-238 & P U-239 \\ M C I & \text { MCI }\end{array}$

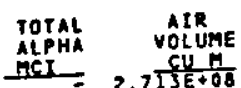

0.016

$0.0028 .141 E+08$

0.050

0.200
IIQUID

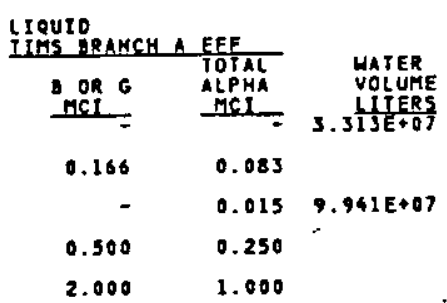

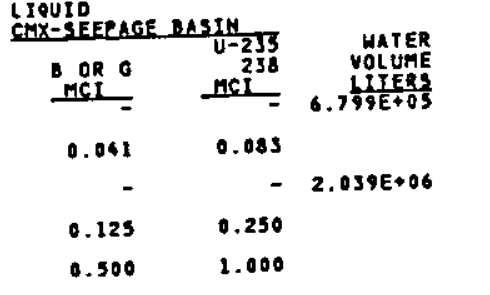

- ACCURACY NOT ALWAYS IMPLIED BY NUMBER OF SIGMIFICANT DIOITS - EXCEEOS PER IOD AND $0 R$ YTO GUIDES MCI 
TABLE HP-31

PLANI STREAMS WATER QUALIIY
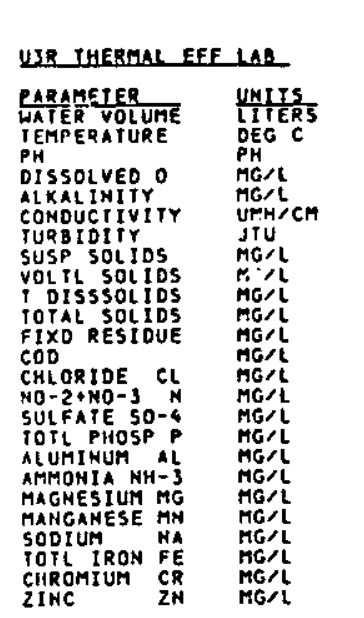
UPPER 3 RUNS ROAD A
WATER VOLUHE LITERS
PERPERATURE DOG C

PH.

DISSOLVED ${ }^{\circ}$ ALKALINITY TUR日DITY SUSP $50 L$ IDS VOLTL SOLIDS TOIS SOLIDS TOTAL SOLIDS FIXD RESIDUE COO CHLORIDE CL NO-2 +NO-3 N SULFATE SO-4 IOTL PHOSP AMMOHIA HA MAGTESTOM HO MANGANESE MH TOIL IROH FE CHROMIUH CR DEG C
PH
HG/L
MG/L
UMH/C
MTU
MG/L
MG/L
MG/L
MG/L
MG/L
MG/L
MG/L
MG/L
MG/L
MG/L
MG/L
MG/L
MG/L
MG/L
MO/L
MG/L
MG/L
$M G / L$

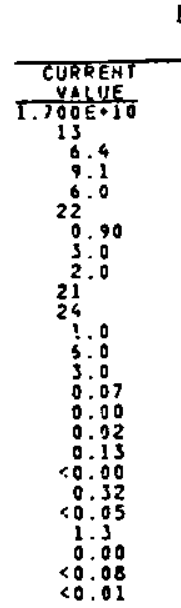
CONCENTRAITOM
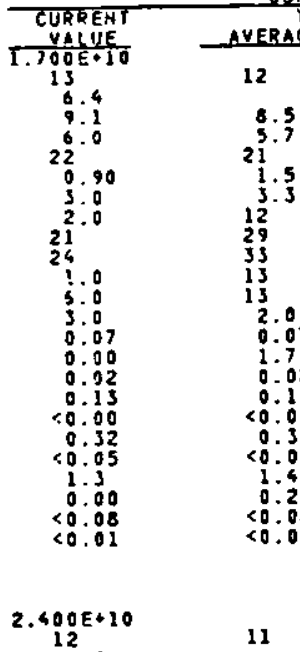

$\begin{array}{ccc}000 E+10 & 11 & (6.200 E+10 \text { TOTAL) } \\ 12 & 14.6 & 6.6 \\ 6.6 & 35 & 88.0 \\ 6.0 & 6.3 & 23.0 \\ 23.0 & 22 & 2.1\end{array}$

5.90

28

$28.0 \quad 13$

30
35
13
15
2.5

0.06

0.00

0.04

0.01
0.32
$<0.05$

$<0.05$

1.5
0.00
$<0.08$

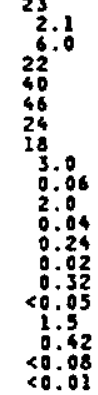

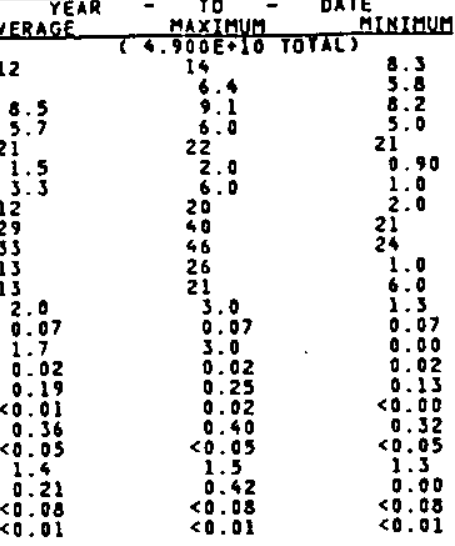

MARCH 31. 1983

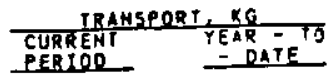

$\begin{array}{ll}1.546 E+05 & 4.186 E+05 \\ 1.019 E+05 & 2.779 E+05\end{array}$

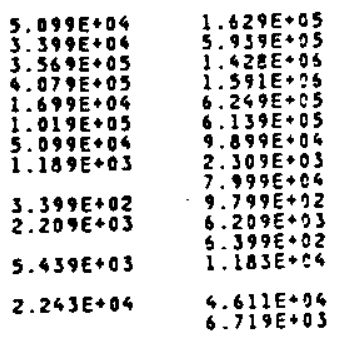

7.7
6.0
8.0
5.0
21.00
0.90
2.0
3.0
23
28
2.0
13.0
1.9
0.06
0.00
0.02
0.18
0.01
0.30
$<0.05$
1.53
0.00
$<0.08$
$<0.01$

$2.111 E+06$
$1.43,+405$

$1.199 E+05 \quad 2.759 E+05$

$7.19 q_{E+0}$

$2.759 E+05$
$8.359 E+05$
$1.855 E+06$

$6.719 E+05 \quad 2.131 E+06$

3.129E+05 $9.059 E+05$

$3.119 E+05 \quad 1.0591 E+05$

$1.439 E+03 \quad 2.639 E+03$

$9.599 E+02 \quad 1.359 E+03$

$2.399 \mathrm{E}+02 \quad 9.99 \mathrm{E}+0$

$2.36,1.04$

3. $599 E+04$

$8.5192+049$

ACCURACY NOT ALWAYS IMPLIED OY MUMBER OF SIGNIFICANT DIGITS 